\title{
AVERAGING AND STABILITY OF QUASILINEAR FUNCTIONAL DIFFERENTIAL EQUATIONS WITH MARKOV PARAMETERS
}

\author{
LAMBROS KATAFYGIOTIS \\ Hong Kong University of Science and Technology \\ Department of Civil and Structural Engineering \\ Clear Water Bay, Kowloon, Hong Kong \\ E-mail: lambros@usthk.ust.hk \\ YEVGENY TSARKOV \\ Riga Technical University \\ Institute of Information Technology \\ 9, Ausekla Iela, Riga LV-1010 Latvia \\ E-mail: carkovs@egle.cs.rtu.lv
}

(Received March, 1997; Revised January, 1998)

\begin{abstract}
An asymptotic method for stability analysis of quasilinear functional differential equations, with small perturbations dependent on phase coordinates and an ergodic Markov process, is presented. The proposed method is based on an averaging procedure with respect to: 1) time along critical solutions of the linear equation; and 2) the invariant measure of the Markov process. For asymptotic analysis of the initial random equation with delay, it is proved that one can approximate its solutions (which are stochastic processes) by corresponding solutions of a specially constructed averaged, deterministic ordinary differential equation. Moreover, it is proved that exponential stability of the resulting deterministic equation is sufficient for exponential $p$-stability of the initial random system for all positive numbers $p$, and for sufficiently small perturbation terms.
\end{abstract}

Key words: Functional Differential Equations, Small Random Perturbations, Stochastic Stability, Averaging Procedures.

AMS subject classifications: $60 \mathrm{H} 10,60 \mathrm{H} 30$.

\section{Introduction}

This paper deals with the $n$-dimensional functional differential equation in a quasilinear form with a small parameter $\varepsilon \in[0,1)$ :

$$
\frac{d u^{\varepsilon}(t)}{d t}=g\left(u_{t}^{\varepsilon}\right)+\varepsilon F\left(t, u_{t}^{\varepsilon}, y(t), \varepsilon\right),
$$

Printed in the U.S.A. C1999 by North Atlantic Science Publishing Company 
where

1) $u_{t}^{\varepsilon}$ is part of the solution defined by the equality $u_{t}^{\varepsilon}:=\left\{u^{\varepsilon}(t+\theta), \theta \in\right.$ $[-h, 0]\}$, with some positive number $h$;

2) $\quad g(\varphi)$ is the linear continuous mapping of the space of the continuous $n$-dimensional vector-functions $\mathbf{C}_{n}:=\mathbf{C}_{n}([-h, 0])$ to $\mathbb{R}^{n}$, defined by the equality:

$$
g(\varphi):=\int_{-h}^{0}\{d G(\theta)\} \varphi(\theta)
$$

with matrix $G(\theta)$ consisting of bounded variation functions;

3) $\{y(t), t \geq 0\}$ is a homogeneous ergodic Markov process on the probability space $(\Omega, \mathcal{F}, \mathbf{P})$, with values in the phase space $\mathbf{Y}$, with infinitesimal operator $Q$, transition probability $P(t, y, d z)$, and unique invariant measure $\mu(d y)$ satisfying the condition of exponential ergodicity (see Blankenship and Papanicolaou [1]). Thus, there exist positive constants $M$ and $\delta$ such that $\|P(t, y, \cdot)-\mu\| \leq M \exp \{-\delta t\}$ for any $t \geq 0$; and

4) the perturbing term $F(t, \varphi, y, \varepsilon)$ is a continuous mapping of the product space $\mathbb{R}_{+} \times \mathbf{C}_{n}([-h, 0]) \times \mathbf{Y} \times[0,1)$ to the space $\mathbb{R}^{n}$, satisfying $F(t, 0, y, \varepsilon) \equiv$ 0 and the Lipschitz condition

$$
|F(t, \varphi, y, \varepsilon)-F(t, \psi, y, \varepsilon)| \leq l \int_{-h}^{0}|\varphi(s)-\psi(s)| d \nu(s)
$$

for any $y \in \mathbf{Y}, \varepsilon \in[0,1), t \in \mathbb{R}_{+}$, and $\varphi, \psi \in \mathbf{C}_{n}$, with some function $\nu(s)$ of unit variation.

Under these conditions, the random Equation (1) with initial problem $u^{\varepsilon}(s+\theta)=$ $\varphi(\theta),-h \leq \theta \leq 0$, has (see Hale and Sjord [4]) a unique solution $u^{\varepsilon}=\left\{u^{\varepsilon}(t), t \geq 0\right\}$ for any continuous function $\varphi$; this solution is a continuous stochastic process with probability one. We will refer to the linear equation:

$$
\frac{d u(t)}{d t}=\int_{-h}^{0}\{d G(\theta)\} u(t+\theta)
$$

as the generative equation corresponding to Equation (1). It is well known (see Hale and Sjord [4]) that Equation (3) defines, in the space $\mathbf{C}_{n}$, a strong continuous semigroup $T(t)$ with infinitesimal operator given for a sufficiently smooth function $\varphi$ by:

$$
(\mathbb{A} \varphi)(\theta):=\left\{\begin{array}{cc}
\frac{d \varphi(\theta)}{d \theta}, & \text { if }-h \leq \theta<0, \\
g(\varphi), & \text { if } \theta=0 .
\end{array}\right.
$$

The spectrum $\sigma(A)$ of this operator is given by:

$$
\sigma(U):=\{z: \operatorname{det}\{U(z)\}=0\}
$$

where 


$$
U(z):=I z-\int_{-h}^{0} e^{z \theta} d G(\theta)
$$

As in the deterministic case (see Halanay [3]), we will proceed in this paper with the assumption that the generative equation is on the border of stability, that is:

$$
\sigma(U) \cap\left\{z: \Re_{0} z>0\right\}=\emptyset, \sigma_{0}:=\sigma(U) \cap\left\{z: \Re_{0} z=0\right\} \neq \emptyset .
$$

We will refer to the spectral subspace of the operator $A$ corresponding to $\sigma_{0}$ as the critical subspace, and to the solutions of Equation (3) lying in the critical subspace as the critical solutions.

Using projection on the critical subspace, we will construct a finite-dimensional differential equation with Markov parameters and rapid switchings, which has the same stability properties of the trivial solution as Equation (1) for all sufficiently small $\varepsilon>0$. It will be proven that for stability analysis under some additional assumptions, one can perform averaging with respect to: 1) the invariant measure of the Markov process; and 2) time along the critical solutions of the generative equation, as one can do for the deterministic delay equations (see Halanay [3]). Stability results can then be obtained applying the Second Lyapunov Method using a specially constructed (see Blankenship and Papanicolaou [1] and Korolyuk [7]) Lyapunov functional and recursive approximations of the solutions of Equation (1) given by the solutions of the corresponding averaged equation.

\section{Result and Discussion}

Some preliminary preparation is needed in order to obtain the resulting averaged equation. First, we rewrite Equation (1) in the operator form (see Hale and Sjord [4]):

$$
\frac{d u_{t}^{\varepsilon}}{d t}=A u_{t}^{\varepsilon}+\varepsilon 1 F\left(t, u_{t}^{\varepsilon}, y(t), \varepsilon\right)
$$

where the matrix-valued function $\{1(\theta),-h \leq \theta \leq 0\}$ is defined by the equality:

$$
\mathbf{1}(\theta):=\left\{\begin{array}{cc}
0, & \text { if }-h \leq \theta<0, \\
I, & \text { if } \theta=0,
\end{array}\right.
$$

and $I$ is the $n \times n$ identity matrix. Next, we define the spectral projective operator $P_{0}$ corresponding to $\sigma_{0} \subset \sigma(A)$. For this, we will use its integral representation (see Kato [5]) in the form:

$$
\left(P_{0} \psi\right)(\theta):=\frac{1}{2 \pi i} \int_{\mathscr{B}^{B}}((J z-A)-1 \psi)(\theta) d z
$$

where $\mathscr{B}:=\bigcup_{j=1}^{m}\left\{z:\left|z-z_{j}\right|=\delta\right\}$ with sufficiently small $\delta>0$. It can be easily seen that both the projective operator $P_{0}$ and $I-P_{0}$ are bounded.

One can apply the projective operator $P_{0}$ not only on any continuous vectorfunction $\psi(\theta)$, but also on any vector- or matrix-valued measurable function. 
Inserting the above matrix-valued function 1 into the integral representation from Equation (5), one can define the $n \times n$-matrix-function:

$$
\left.\Gamma(\theta):=\frac{1}{2 \pi i} \int_{\mathscr{B}}((J z-A))^{-1} 1\right)(\theta) d z=\left.\sum_{j=1}^{m} \operatorname{res}\left\{U^{-1}(z) e^{z \theta}\right\}\right|_{z=z_{j}}
$$

Let us denote the critical subspace as $\mathbf{X}_{0}:=P_{0} \mathbf{C}_{n}$, the $n \times m$-matrix of a basis in this subspace as $V(\theta)$, the restriction of the operator $A$ on $\mathbf{X}_{0}$ as $\mathbb{A}_{0}$, and let $A_{0}$ be the matrix of this restriction, as defined by the equation $A_{0} V(\theta)=V(\theta) A_{0}$. Furthermore, one can define the $m \times m$-matrix $\widehat{\Psi}$, writing the identity $\Gamma(\theta)=V(\theta) \widehat{\Psi}$. Let us use the above notations, along with the notation $\mathbf{V}:=\{V(\theta),-h \leq \theta \leq 0\}$, and assume the existence of the $m$-dimensional vector function $\check{F}(x)$ of argument $x \in \mathbb{R}^{m}$ defined by:

$$
\check{F}(x):=\lim _{T \rightarrow \infty} \frac{1}{T} \int_{0}^{T} \int_{\mathbf{Y}} e^{-t A_{0}} \widehat{\Psi} F\left(t, \mathbf{V} e^{t A_{0}} x, y, 0\right) \mu(d y) d t
$$

where $\mu(d y)$ is the invariant measure of the Markov process $y(t), t \geq 0$. Thus, we define the averaged differential equation (which is not random):

$$
\frac{d \check{x}}{d t}=\check{F}(\check{x}) \text {. }
$$

We say that the trivial solution of Equation (7) is exponentially stable in the large if there exist positive constants $\check{a}_{1}$ and $\check{a}_{2}$ such that:

$$
|\check{x}(t+s, s, x)| \leq \check{a}_{1} e^{-\check{a}_{2} t}|x|
$$

for any $s, t \geq 0, x \in \mathbb{R}^{m}$. We say that the trivial solution of the random Equation (1) is exponentially $p$-stable in the large for all sufficiently small positive $\varepsilon$ if there exist positive constants $\varepsilon_{0}, a_{1}$, and for any $\varepsilon \in\left(0, \varepsilon_{0}\right)$, there exists a positive number $a_{2}(\varepsilon)$ such that:

$$
\mathbf{E}_{y, \varphi}^{(s)}\left\{\left|u^{\varepsilon}(t+s)\right|^{p}\right\} \leq a_{1} e^{-a_{2}(\varepsilon) t}\|\varphi\|^{p}
$$

for any $s, t \geq 0, y \in \mathbf{Y}$, and $\varphi \in \mathbf{C}_{n}$. In this definition and throughout this paper, the above upper and lower indices of expectation (or probability) denote the conditions $y(s)=y, u_{s}^{\varepsilon}=\varphi$. All subsequent relations involving random variables and processes are understood as such.

The selection of the linear mapping $g(\varphi)$ in the right part of Equation (1) can be accomplished somewhat arbitrarily by adding any arbitrary linear continuous mapping $\varepsilon g_{1}(\varphi)$ to the linear part of Equation (1) and subtracting it from the second term. Because the set $\sigma_{0}$ consists of a finite number of points (see Hale and Sjord [4]) $\sigma_{0}=$ $\left\{z_{j}, j=1,2, \ldots, m\right\}$, it may be assumed that the selection of terms in the right part of Equation (1) has been done in such a manner so that $\left(\operatorname{det} U\left(z_{j}\right)\right)^{\prime} \neq 0, j=1,2, \ldots, m$.

Lemma 1: Under the above assumptions, one can find a constant $c$ such that the solution of Equation (1) with initial condition:

satisfies the inequality:

$$
u^{\varepsilon}(s+\theta)=\varphi(\theta), \quad-h \leq \theta \leq 0
$$




$$
\sup _{\substack{0 \leq t \leq T / \varepsilon \\ s \geq 0, y \in \mathbf{Y}}}\left|u^{\varepsilon}(t+s, s, \varphi)\right| \leq c e^{c l T}\|\varphi\|
$$

for any $T>0, \varepsilon \in(0,1)$, where $l$ is the Lipschitz constant from Equation (2).

Proof: Let $H(t)$ denote the matrix-solution of the generative Equation (3) satisfying the initial condition:

$$
H(\theta)=1(\theta),-h \leq \theta \leq 0
$$

Using this matrix-valued function, one can (see Hale and Sjord [4]) rewrite Equation (1) in the integral form:

$$
u^{\varepsilon}(t+s, s, \varphi)=u^{0}(t, 0, \varphi)+\varepsilon \int_{0}^{t} H(t-\tau) F\left(s+\tau, u_{s+\tau}^{\varepsilon}, y(s+\tau), \varepsilon\right) d \tau
$$

where $u^{0}(t, 0, \varphi)$ is the solution of the generative equation with the same initial condition. Due to our assumptions regarding the spectrum part $\sigma_{0}$, there exists (see Hale and Sjord [4]) $c:=\sup _{t \geq 0}\|T(t)\|$, whence:

$$
\|H(t)\| \leq \operatorname{csup}_{h \leq \theta \leq 0}\|\mathbf{1}(\theta)\| \leq c,\left|u^{0}(t, 0, \varphi)\right|=|(T(t) \varphi)(0)| \leq c\|\varphi\|,
$$

for any $t \geq 0$ and $\varphi \in \mathbf{C}_{n}$. Therefore, the proof follows from the integral inequality

$$
\sup _{t_{1} \leq t}\left|u^{\varepsilon}\left(t_{1}+s, s, \varphi\right)\right| \leq c\|\varphi\|+\varepsilon l c \int_{0}^{t} \sup _{t_{1} \leq \tau}\left|u^{\varepsilon}\left(t_{1}+s, s, \varphi\right)\right| d \tau
$$

after applying the Gronwall's lemma on the segment $0 \leq t \leq T / \varepsilon$.

Using the matrix $\Gamma(\theta)$ from Equation (6) and the decomposition:

$$
u_{t}^{\varepsilon}(\theta)=\left(P_{0} u_{t}^{\varepsilon}\right)(\theta)+\left(\left(I-P_{0}\right) u_{t}^{\varepsilon}\right)(\theta)
$$

one can rewrite Equation (4) as a system of two equations for the vector-functions $r_{0}(t, \theta):=\left(P_{0} u_{t}^{\varepsilon}\right)(\theta)$ and $r_{1}(t, \theta):=\left(\left(I-P_{0}\right) u_{t}^{\varepsilon}\right)(\theta)$ :

$$
\begin{aligned}
& \frac{\partial r_{0}(t, \theta)}{\partial t}=\left(\mathbb{A}_{0} r_{0}\right)(t, \theta)+\varepsilon \Gamma(\theta) F\left(t, \mathbf{r}_{\mathbf{0}}(t)+\mathbf{r}_{\mathbf{1}}(t), y(t), \varepsilon\right), \\
& \frac{\partial r_{1}(t, \theta)}{\partial t}=\left(\mathbb{A}_{1} r_{1}\right)(t, \theta)+\varepsilon(1(\theta)-\Gamma(\theta)) F\left(t, u_{t}^{\varepsilon}, y(t), \varepsilon\right),
\end{aligned}
$$

where $\mathbf{r}_{j}(t)=\left\{r_{j}(t, \theta), \theta \in[-h, 0]\right\}, j=0,1$, and the linear closed operator $\mathbb{A}_{1}:=$ $\left(J-P_{0}\right) \mathbb{A}$ is acting on the same subspace $\mathscr{D}(\mathbb{A}) \subset \mathbf{C}_{n}$ as the operator $\mathbb{A}$, and has the spectrum $\sigma_{1}:=\sigma(U) \backslash \sigma_{0} \subset\left\{\Re_{0} z \leq-\rho<0\right\}$.

Lemma 2: Under the above conditions there exists constant $c_{1}$ such that the solution of Equation (12) satisfies the inequality:

$$
\begin{aligned}
& \sup _{\substack{s \geq 0, y \in \mathbf{Y} \\
-h \leq \theta \leq 0}}\left|r_{1}(t, \theta)-\left(T(t)\left(I-P_{0}\right) \varphi\right)(\theta)\right| \leq \varepsilon c_{1}\|\varphi\| e^{l c T} \\
& 0 \leq t \leq T / \varepsilon
\end{aligned}
$$


for any $T>0, \varepsilon \in(0,1)$ with constant c from Equation (10).

Proof: The operator $A_{1}$ can be considered (see Kato [5]) as the infinitesimal operator of the contractive semigroup $\left\{T_{1}(t), t \geq 0\right\}$, which satisfies the inequality:

$$
\left\|T_{1}(t)\right\| \leq \gamma e^{-\rho t}
$$

with the above defined positive $\rho$ and some positive constant $\gamma$ for any $t \geq 0$. Using this semigroup, one can rewrite Equation (12) in the integral form

$$
r_{1}(s+t, \theta)=\left(T(t)\left(I-P_{0}\right) \varphi\right)(\theta)+\varepsilon \int_{s}^{s+t} T_{1}(t-\tau)(1(\theta)-\Gamma(\theta)) F\left(\tau, u_{\tau}^{\varepsilon}, y(\tau), \varepsilon\right) d \tau
$$

Due to Lemma 1, Equation (10), the Lipschitz condition and the exponential decay of the semigroup $T_{1}(t)$ the above integral equality allows us to complete the proof using the inequality:

$$
\sup _{\substack{0 \leq t \leq T / \varepsilon \\ s \geq 0, y \in \mathbf{Y}}}\left\|\mathbf{r}_{\mathbf{1}}(t+s)-T(t)\left(I-P_{0}\right) \varphi\right\| \leq \varepsilon\left(1+\left\|P_{0}\right\|\right) \frac{l c \gamma}{\rho} e^{l c T}\|\varphi\|
$$

or

$$
\sup _{s \geq 0, y \in \mathbf{Y}}\left\|\mathbf{r}_{\mathbf{1}}(T / \varepsilon+s)\right\| \leq \varepsilon \gamma_{1} e^{l c T}\|\varphi\|+\gamma e^{-\frac{t \rho}{\varepsilon}}
$$

for any $T>0, \varphi \in(0,1), \varepsilon \in \mathbf{C}_{n}$, and with $\gamma_{1}$ being a positive constant.

Theorem 1: Let, in addition to the previous assumptions, the function $F(t, \mathrm{~V} x, y, \varepsilon)$ be uniformly continuous at zero as a function of $\varepsilon$, that is, assume that the quantity

$$
\alpha(\varepsilon): \sup _{\substack{t \geq 0, y \in \mathbf{y} \\ x \in \mathbb{R}^{n}}} \frac{|F(t, \mathbf{V} x, y, \varepsilon)-F(t, \mathbf{V} x, y, 0)|}{|x|}
$$

is infinitesimal as $\varepsilon \rightarrow 0$, and the limit function $F(t, \mathbf{V} x, y, 0)$ :

1) has uniformly bounded continuous $x$-derivative $D F(t, \mathbf{V} x, y, 0)$;

2) belongs to the domain $\mathfrak{D}(Q)$ of the operator $Q$;

3) has continuous bounded $t$-derivative $\frac{\partial}{\partial t} F(t, \mathbf{V} x, y, 0)$;

4) has the above defined average $\breve{F}(x)$ along the solutions of the generative equation, and there exists constant $b$ such that:

$\sup _{y \in \mathbf{Y}, T>0}\left|\int_{s \geq 0}^{s+T} \int_{\mathbf{Y}} e^{-t A_{0}} \widehat{\Psi} F\left(t, \mathbf{V} e^{t A_{0}} x, y, 0\right) \mu(d y) d t-T \bar{F}(x)\right| \leq b|x|$,

for any $x \in \mathbb{R}^{m}$.

If the trivial solution of the averaged Equation (7) is exponentially stable in the large, then the trivial solution of the random Equation (1) is exponentially p-stable in the large for all sufficiently small positive $\varepsilon$.

Proof: According to the definition of the basis $V(\theta)$, the $n$-dimensional vectorfunction $r_{0}(t, \theta)$ in Equation (11) can be decomposed as follows: 


$$
r_{0}(t, \theta):=V(\theta) \vec{u}^{\varepsilon}(t), \quad \forall \theta \in[-h, 0]
$$

After substitution of this decomposition in Equation (11), one can conclude that the $m$-dimensional vector-function $\vec{u}^{\varepsilon}(t)$ satisfies the ordinary random differential equation in $\mathbb{R}^{m}$

$$
\frac{d \vec{u}^{\epsilon}(t)}{d t}=A_{0} \vec{u}^{\varepsilon}(t)+\varepsilon \widehat{\Psi} F\left(t, \mathbf{V} \vec{u}^{\varepsilon}(t)+\mathbf{r}_{\mathbf{1}}(t), y(t), \varepsilon\right) .
$$

One can consider the decomposition of Equation (1) in the forms of Equations (11) and (12) with the decomposed initial condition in Equation (9):

$$
r_{0}(s, \theta)=P_{0} \varphi(\theta):=V(\theta) \vec{u}, \quad r_{1}(s, \theta)=\left(I-P_{0}\right) \varphi(\theta)
$$

which uniquely defines the vector $\vec{u} \in \mathbb{R}^{m}$ for given basis V. Consequently, Equation (16) should be considered with initial condition:

$$
\vec{u}^{\varepsilon}(s)=\vec{u}
$$

Let $\tilde{u}^{\varepsilon}(t)$ be the solution of the random differential equation in $\mathbb{R}^{m}$

$$
\frac{d \widetilde{u}^{\varepsilon}(t)}{d t}=A_{0} \tilde{u}^{\varepsilon}(t)+\varepsilon \widehat{\Psi} F\left(t, \mathbf{V} \tilde{u}^{\varepsilon}(t), y(t), \varepsilon\right)
$$

with the above initial condition to Equation (18). Using the substitutions

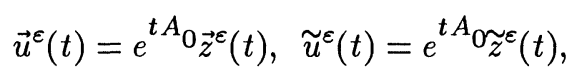

one can derive from Equation (16), for the vector-valued functions $\vec{z}^{\varepsilon}(t)$ and $\widetilde{z}^{\varepsilon}(t)$, the equations

$$
\begin{gathered}
\frac{d \vec{z}^{\varepsilon}(t)}{d t}=\varepsilon e^{-t A_{0}} \widehat{\Psi} F\left(t, \mathbf{V} e^{\left.t A_{0} \vec{z}^{\varepsilon}(t)+\mathbf{r}_{\mathbf{1}}(t), y(t), \varepsilon\right)}\right. \\
\frac{d \widetilde{z}^{\varepsilon}(t)}{d t}=\varepsilon e^{-t A_{0}} \widehat{\Psi} F\left(t, \mathbf{V} e^{t A_{0} \widetilde{z}^{\varepsilon}}(t), y(t), \varepsilon\right)
\end{gathered}
$$

Due to the Lipschitz condition in Equation (2) and the assumptions regarding the spectrum $\sigma_{0}$ of the matrix $A_{0}$, the difference of the solutions satisfies the integral inequality

$$
\begin{aligned}
& \left|\vec{z}^{\varepsilon}(t+s)-\widetilde{z}^{\varepsilon}(t+s)\right| \leq \varepsilon c l\|\widehat{\Psi}\| \int_{0}^{t} \int_{-h}^{0}\|V(\theta)\|\left|r_{1}(\tau+s, \theta)\right| d \nu(\theta) d \tau \\
& \quad+\varepsilon c l\|\widehat{\Psi}\| \int_{-h}^{0}\|V(\theta)\| d \nu(\theta) \int_{0}^{t}\left|\vec{z}^{\varepsilon}(\tau+s)-\widetilde{z}^{\varepsilon}(\tau+s)\right| d \tau .
\end{aligned}
$$

Using the inequality in Equation (13), one can derive the inequality

$$
\left\|\mathbf{r}_{\mathbf{1}}(\tau+s)\right\| \leq \gamma e^{-\rho \tau}\left(1+\left\|P_{0}\right\|\right)\|\varphi\|+\varepsilon c_{1} e^{l c T}\|\varphi\|,
$$

for any $T>0, \tau \in[0, T / \varepsilon)$. Therefore, there exist a constant $l_{1}$ and a function $l_{2}(T)$ 
such that the difference between the solutions of Equations (20) and (21) satisfies the inequality

$$
\left|\vec{z}^{\varepsilon}(t+s)-\widetilde{z}^{\varepsilon}(t+s)\right| \leq \varepsilon l_{2}(T)\|\varphi\|+\varepsilon l_{1} \int_{0}^{t}\left|\vec{z}^{\varepsilon}(\tau+s)-\widetilde{z}^{\varepsilon}(\tau+s)\right| d \tau .
$$

Applying Gronwall's inequality, one can find a function $c_{3}(T)$ such that:

$$
\sup _{\substack{0 \leq t \leq T / \varepsilon \\ s \geq 0, y \in \mathbf{Y}}}\left|\vec{z}^{\varepsilon}(t+s)-\widetilde{z}^{\varepsilon}(t+s)\right| \leq \varepsilon c_{3}(T)\|\varphi\|
$$

for any $T>0, \varepsilon \in[0,1), \varphi \in \mathbf{C}_{n}$.

To simplify the notations, we denote

$$
\tilde{x}^{\varepsilon}(t):=\tilde{z}^{\varepsilon}(t / \varepsilon), f(t, x, y, \varepsilon):=e^{-t A_{0}} \widehat{\Psi} F\left(t, \mathbf{V} e^{t A_{0}} x, y, \varepsilon\right) .
$$

Let $x^{\varepsilon}(t)$ be the solution of the random equation

$$
\frac{d x^{\varepsilon}}{d t}=f\left(t / \varepsilon, x^{\varepsilon}, y(t / \varepsilon), 0\right) .
$$

It is easy to verify that $\tilde{x}^{\varepsilon}(t)$ satisfies the random differential equation:

$$
\frac{d \widetilde{x}^{\varepsilon}}{d t}=f\left(t / \varepsilon, \widetilde{x}^{\varepsilon}, y(t / \varepsilon), \varepsilon\right) .
$$

Consider this equation with initial conditions $\tilde{x}^{\varepsilon}(s)=x^{\varepsilon}(s)=\vec{u}$, with vector $\vec{u}$ from Equation (17). Due to the existence of the uniformly bounded $x$-derivative $D F(t, \mathrm{~V} x, y, 0)$, the right-hand sides of Equations (23) and (24) satisfy the Lipschitz condition with some constant $L$. Furthermore, it follows from Equation (14) that the function $f(t, x, y, \varepsilon)$ is uniformly continuous at point zero as a function of $\varepsilon$, that is, the quantity

$$
\beta(\varepsilon): \sup _{\substack{t \geq 0, y \in \mathbf{y} \\ x \in \mathbb{R}^{n}}} \frac{|f(t, x, y, \varepsilon)-f(t, x, y, 0)|}{|x|}
$$

is infinitesimal as $\varepsilon \rightarrow 0$. Using the latter property and the Lipschitz constant $L$, one can write the inequalities

$$
\begin{gathered}
\left|\tilde{x}^{\varepsilon}(t+s, s, \vec{u})-x^{\varepsilon}(t+s, s, \vec{u})\right| \\
\leq \int_{s}^{s+t}\left|f\left(\tau / \varepsilon, \widetilde{x}^{\varepsilon}(\tau, s, \vec{u}), y(\tau / \varepsilon), \varepsilon\right)-f\left(\tau / \varepsilon, x^{\varepsilon}(\tau, s, \vec{u}), y(\tau / \varepsilon), 0\right)\right| d \tau \\
\leq L \int_{0}^{t}\left|\tilde{x}^{\varepsilon}(\tau+s, s, \vec{u})-x^{\varepsilon}(\tau+s, s, \vec{u})\right| d \tau \\
+\int_{s}^{s+t}\left|f\left(\tau / \varepsilon, x^{\varepsilon}(\tau, s, \vec{u}), y(\tau / n), \varepsilon\right)-f\left(\tau / \varepsilon, x^{\varepsilon}(\tau, s, \vec{u}), y(\tau / \varepsilon), 0\right)\right| d \tau
\end{gathered}
$$




$$
\leq L \int_{0}^{t}\left|\widetilde{x}^{\varepsilon}(\tau+s, s, \vec{u})-x^{\varepsilon}(\tau+s, s, \vec{u})\right| d \tau+\beta(\varepsilon) \int_{s}^{s+t}\left|x^{\varepsilon}(\tau, s, \vec{u})\right| d \tau .
$$

It can be easily shown that the Lipschitz condition for the right-hand side of Equation (23) guarantees the existence of a constant $B$, such that:

$$
\left|x^{\varepsilon}(t+s, s, \vec{u}) \leq B e^{L t}\right| \vec{u} \mid
$$

for any $\vec{u} \in \mathbb{R}^{m}, s \geq 0, t \geq 0, \varepsilon \in[0,1)$. Thus, substituting this bound in the last term of Equation (25) and applying Gronwall's inequality, one can obtain the relation

$$
\sup _{\substack{0 \leq t \leq T \\ s \geq 0, y \in \mathbf{Y}}}\left|\tilde{x}^{\varepsilon}(t+s, s, \vec{u})-x^{\varepsilon}(t+s, s, \vec{u})\right| \leq \beta(\varepsilon) B T e^{L T}|\vec{u}|,
$$

for any $T \geq 0, \vec{u} \in \mathbb{R}^{m}$ and sufficiently small $\varepsilon>0$. For further analysis, it is convenient to rewrite this inequality for the time $t \varepsilon$ and use the norm of the initial condition in Equation (9):

$$
\sup _{\substack{0 \leq t \leq T / \varepsilon \\ s \geq 0, y \in \mathbf{Y}}}\left|\widetilde{x}^{\varepsilon}(t \varepsilon+s, s, \vec{u})-x^{\varepsilon}(t \varepsilon+s, s, \vec{u})\right| \leq \beta(\varepsilon) B T e^{L T}\|\varphi\|,
$$

for any $T \geq 0, \varphi \in \mathbf{C}_{n}$.

It is known (see Blankenship and Papanicolaou [1] and Skorokhod [9]) that under the above assumptions, the solutions of Equation (24) tend to the corresponding solutions of Equation (7), and that the stability of the trivial solution of Equation (7) guarantees (see Korolyuk [7]) the stability with probability one of the trivial solutions of Equation (24). However, in order to prove our theorem, we need stronger evaluation of the rate of convergence to zero of the $p$-moments of the solutions of Equation (24) as $t \rightarrow \infty$. For this purpose, we will apply the second Lyapunov method to a specially constructed functional $v(t, x, y, \varepsilon)$. Since for any random variable $\xi$, the quantity $\left(\mathbb{E}\left(|\xi|^{p}\right)\right)^{1 / p}$ is monotonically nondecreasing function of $p>0$, we can assume in our proof without loss of generality that $p \geq 2$.

One can consider the pair $\left\{x^{\varepsilon}, y(t / \varepsilon)\right\}$ as a Markov process in the phase space $\mathbb{R}^{m} \times \mathbf{Y}$ (see Blankenship and Papanicolaou [1], Mohammed [8], and Skorokhod [9]) with weak infinitesimal operator $\mathbb{Q}$ defined on sufficiently smooth continuous function $v(x, y)$ by the equality:

$$
(\mathcal{Q} v)(x, y):=(\nabla v)(x, y)+\frac{1}{\varepsilon}(Q v)(x, y)
$$

where $(\cdot, \cdot)$ and $\nabla$ are the scalar product and the gradient-operator in $\mathbb{R}^{m}$, respectively. Since the right-hand side of Equation (23) depends on the time coordinate $t$, one needs to extend the phase space by adding a new phase coordinate $t \in \mathbb{R}+$, and consider the above nonhomogeneous Markov process with infinitesimal operator:

$$
\begin{gathered}
(\ell v)(t, x, y):=\frac{1}{\varepsilon}\left(\left(\frac{\partial}{\partial t} v\right)(t, y, x)+(Q v)(t, x, y)\right) \\
+((\nabla v)(t, x, y), f(t / \varepsilon, x, y, 0)) .
\end{gathered}
$$

For a given function $w(t, y)$, let $\widehat{w}(t)$ denote the function obtained by averaging 
$w(t, y)$ with respect to the invariant measure $\mu(d y)$, that is,

$$
\widehat{w}(t):=\int_{\mathbf{Y}} w(t, y) \mu(d y),
$$

and let $\bar{w}(y)$ denote the function obtained by averaging $w(t, y)$ with respect to the time $t$, that is,

$$
\bar{w}(y):=\lim _{T \rightarrow \infty} \frac{1}{T} \int_{0}^{T} w(t, y) d t .
$$

The projection operator $\mathbf{P}_{\mu}$ on the subspace

$$
\mathbf{C}_{\mu}:=\{g \in \mathbf{C}(\mathbf{Y}): \widehat{g}=0\}
$$

can be defined by the equality $\mathbf{P}_{\mu} g(y):=g(y)-\widehat{g}$. Due to our assumption of exponential ergodicity, we can use the inequality:

$$
\sup _{y \in \mathbf{Y}} \mid \mathbf{E}_{y}\left(\mathbf{P}_{\mu} g\right)\left(y(t)\left|\leq M e^{-\rho t} \sup _{y \in \mathbf{Y}}\right| g(y) \mid\right.
$$

for any $t \geq 0$ and $g \in \mathbf{C}(\mathbf{Y})$ and, therefore, the potential $\prod$ of the Markov process $\{y(t)\}$ can be defined as the improper integral:

which satisfies,

$$
\left(\prod g\right)(y):=\int_{0}^{\infty} \mathbf{E}_{y} g(y(t)) d t
$$

$$
\sup _{y \in \mathbf{Y}}|(\Pi g)(y)| \leq \frac{M}{\delta} \sup _{y \in \mathbf{Y}}|g(y)|
$$

for any $g \in \mathbf{C}_{\mu}$. According to the definition of the weak infinitesimal operator (see Dynkin [2]), one can write the equality:

$$
\frac{\partial}{\partial t} \mathbf{E}_{y} h(s, y(s-t))=-\mathbf{E}_{y}(Q h)(s, y(s-t)),
$$

for any $s>t \geq 0$ and continuous bounded function $h(s, y)$. If in addition, $\widehat{h}(s) \equiv 0$, then the inequality:

$$
\left|\mathbf{E}_{y} h(s, y(s-t))\right| \leq M e^{-\delta(s-t)} \sup _{y \in \mathbf{Y}}|h(s, y)|
$$

follows from Equation (28). Therefore, there exists the improper integral:

$$
\int_{t}^{\infty} \mathbf{E}_{y} h(s, y(s-t)) d s:=G(h)(t, y)
$$

for any $y \in \mathbf{Y}$, and

$$
\sup _{\substack{y \in \mathbf{Y} \\ s \geq 0}}|(G h)(t, y)| \leq \frac{M}{\delta} \sup _{\substack{y \in \mathbf{Y} \\ s \geq 0}}|h(s, y)|
$$


In view of Equation (29), one can easily verify that the function $r(t, y):=G(h)(t, y)$ satisfies the ordinary differential equation:

$$
\frac{d}{d t} r(t, y)+Q r(t, y)=-h(t, y) \text {. }
$$

Using this result and the representation $h(t, y)=(h(t, y)-\widehat{h}(t))+\widehat{h}(t)$, one can find a solution of Equation (31) for arbitrary bounded function $h(t, y)$ in the form:

$$
R(h)(t, y):=G(h-\widehat{h})(t)+\int_{0}^{t} \widehat{h}(s) d s,
$$

and from inequality in Equation (30) obtain the inequality:

$$
|R(h)| \leq 2 \frac{M}{\delta} \sup _{t \geq 0, y \in \mathbf{Y}}|h(t, y)|+\sup _{t \geq 0}\left|\int_{0}^{t} \widehat{h}(s) d s\right| .
$$

To prove the exponential $p$-stability of the trivial solution of Equation (24), we will use the Lyapunov functional:

where

$$
w(t, x, y, \varepsilon):=v(x)+\varepsilon v_{1}(t / \varepsilon, x, y)
$$

$$
v_{1}(t, x, y)=(\nabla v(x), R(f-\check{F})(t, x, y, 0))
$$

and the operator $R$ acts on the function $f(t, x, y, 0)-\check{F}(x)$ according to arguments $t$ and $y$ as defined in Equation (32). The inequalities in Equations (15) and (33) allow us to estimate the second term in the latter scalar product as follows:

$$
|R(f-\check{F})(t, x, y, 0)| \leq 2 \sup _{t \geq 0}\left|\int_{0}^{t} \widehat{h}(s) d s\right|+2 \frac{M}{\delta} \sup _{\substack{y \in \mathbf{Y} \\ t \geq 0}}|f(t, x, y, 0)| .
$$

It is obvious that under the assumption of exponential stability conditions in the large of Equation (7), the $p$ th power of the absolute value of any solution of Equation (7) decreases also exponentially when $t \rightarrow \infty$ for any $p>0$. Therefore, one can consider the function:

$$
v(x)=\int_{0}^{S}|x(t, 0, x)|^{p} d t
$$

with sufficiently large positive $S$ as the Lyapunov function for the averaged system. Using the smoothness with respect to $\check{x}$ of the right-hand side of Equation (7), one can prove that $v(x)$ has continuous derivative $\nabla v(x)$, and that the following inequalities are satisfied:

$$
\begin{gathered}
v_{1}|x|^{p} \leq v(x) \leq v_{2}|x|^{p}, \quad(\nabla v(x), \check{F}(x)) \leq-v_{3}|x|^{p} \\
\sup _{t \geq 0, y \in \mathbf{Y}}\left|v_{1}(t, x, y)\right| \leq v_{4}|x|^{p}, \sup _{t \geq 0, y \in \mathbf{Y}}\left|\nabla v_{1}(t, x, y)\right| \leq v_{4}|x|^{p-1}
\end{gathered}
$$


for any $x \in \mathbb{R}^{m}$ with some positive numbers $v_{1}, v_{2}, v_{3}, v_{4}, v_{5}$. Therefore, for sufficiently small positive $\varepsilon_{1}$, one can write the inequalities:

$$
w_{1}|x|^{p} \leq w(t, x, y, \varepsilon) \leq w_{2}|x|^{p}
$$

with some positive number $w_{1}$ for all $\varepsilon \in\left[0, \varepsilon_{1}\right)$ and arbitrary values of the remaining variables involved in Equation (36). Furthermore, using the definitions in Equations (27) and (32) of the operators $\mathcal{L}$ and $R$, respectively, one can obtain for the quantity $\ell w$ the following inequality:

$$
\begin{gathered}
(\mathcal{w})(t, x, y, \varepsilon):=((\nabla v)(x), f(t / \varepsilon, x, y, 0))\left(\left(\frac{\partial}{\partial t} v_{1}\right)(t / \varepsilon, x, y)\right. \\
\left.\left.+\left(Q v_{1}\right)(t / \varepsilon, x, y)\right)+\varepsilon\left(\nabla v_{1}\right)(t / \varepsilon, x, y), f(t / \varepsilon, x, y, 0)\right) \\
\left.=((\nabla v)(x), \check{F}(x))+\varepsilon\left(\nabla v_{1}\right)(t / \varepsilon, x, y), f(t / \varepsilon, x, y, 0)\right) \\
\leq-w_{3}|x|^{p}
\end{gathered}
$$

for sufficiently small values of $\varepsilon$. Let us assume that $\varepsilon_{1}$ has been chosen small enough so that both of the inequalities in Equations (36) and (37) are fulfilled simultaneously. Then, using the well known Dynkin formula (see Dynkin [2]), and the inequalities in Equations (36) and (37), one can obtain the inequality:

$$
\begin{gathered}
\left.\mathbf{E}\left\{\left|x^{\varepsilon}(t)\right|^{p} \mid x^{\epsilon}(s)=\vec{u}, y(s / \varepsilon)=y\right)\right\} \\
\left.\leq \frac{1}{w_{1}} \mathbf{E}\left\{w\left(t, x^{\epsilon}(t), y(t / \varepsilon), \varepsilon\right) \mid x^{\varepsilon}(s)=\vec{u}, y(s / \varepsilon)=y\right)\right\} \\
=\frac{1}{w_{1}}\left\{w(t, x, y, \varepsilon)+\int_{s}^{t} \mathbf{E}\left\{\ell w\left(\tau, x^{\varepsilon}(\tau), y(\tau / \varepsilon, \varepsilon) \mid x^{\varepsilon}(s)=\vec{u}, y(s / \varepsilon)\right\} d \tau\right\} .\right.
\end{gathered}
$$

Therefore, the conditional $p$-moment of the solution of Equation (23) satisfies the inequality:

$$
\begin{gathered}
\left.\mathbf{E}\left\{\left|x^{\varepsilon}(t)\right|^{p} \mid x^{\varepsilon}(s)=\vec{u}, y(s / \varepsilon)=y\right)\right\} \\
\leq \frac{w_{2}}{w_{1}}|x|^{p}-\frac{w_{3}}{w_{1}} \int_{s}^{t} \mathbf{E}\left\{\left|x^{\varepsilon}(\tau)\right|^{p} \mid x^{\varepsilon}(s)=\vec{u}, y(s / \varepsilon)\right\} d \tau
\end{gathered}
$$

whence one can conclude that:

$$
\left.\mathbf{E}\left\{\left|x^{\varepsilon}(t)\right|^{p} \mid x^{\varepsilon}(s)=\vec{u}, y(s / \varepsilon)=y\right)\right\} \leq \beta_{1}|\vec{u}|^{p} e^{-\beta_{1}(t-s)},
$$

for any $t \geq s \geq 0, \vec{u} \in \mathbb{R}^{m}$ and $\varepsilon \in\left(0, \varepsilon_{1}\right)$, with some positive constant $\beta_{1}$. Using this inequality, one can evaluate the rate of decay of the second moment of the supremum of the solution of Equation (23) in the time-interval [ $-h \varepsilon, 0]$ from:

$$
\left.\mathbf{E}\left\{\sup _{t-h \varepsilon \leq \tau \leq t}\left|x^{\varepsilon}(\tau+s)\right|^{2} \mid x^{\varepsilon}(s)=\vec{u}, y(s / \varepsilon)=y\right)\right\}
$$




$$
\leq|\vec{u}|^{p}+h \varepsilon \int_{t-h \varepsilon}^{t} \beta_{1}|\vec{u}|^{p} e^{-\beta_{1} \tau} d \tau \leq \beta_{2}|\vec{u}|^{p}
$$

for any $t \geq h \varepsilon, x \in \mathbb{R}^{m}, y \in \mathbf{Y}$, and $\varepsilon \in\left(0, \varepsilon_{1}\right)$. It can be easily seen that using this formula, the previous inequality can be rewritten in the form:

$$
\left.\sup _{\substack{s \geq 0 \\ y \in \mathbf{Y}}} \mathbf{E}_{\boldsymbol{y}, \vec{u}}^{s}\left\{\sup _{t-h \varepsilon \leq \tau \leq t}\left|x^{\varepsilon}(\tau+s)\right|^{p} \mid x^{\varepsilon}(s)=\vec{u}, y(s / \varepsilon)=y\right)\right\} \leq \beta_{2}|\vec{u}|^{p} e^{-\beta_{1} t} .
$$

Since the initial condition $\vec{u}$ of Equation (23) is the projection of the initial condition in Equation (9), it follows from the above inequality that:

$$
\begin{gathered}
\sup _{\substack{s \geq 0, y \in \mathbf{Y}}} \mathbf{E}_{y, \varphi}^{s}\left\{\sup _{t-h \varepsilon \leq \tau \leq t}\left|x^{\varepsilon}(\tau+s)\right|^{p}\right\} \\
\left.\sup _{\substack{s \geq 0, y \in \mathbf{Y}}} \mathbf{E}\left\{\sup _{t-h \varepsilon \leq \tau \leq t}\left|x^{\varepsilon}(\tau+s)\right|^{p} \mid u^{\varepsilon}(s)=\varphi, y(s / \varepsilon)=y\right)\right\} \\
\leq \beta_{3}\|\varphi\|^{p} e^{-\beta_{1} t}
\end{gathered}
$$

for any $\varphi \in \mathbf{C}_{n}$.

By construction the solution of Equations (1) and (9) satisfies the inequalities:

$$
\begin{aligned}
& \sup _{\substack{s \geq 0, y \in \mathbf{Y}}}\left\|u_{t+s}^{\varepsilon}(s, \varphi)\right\| \leq \sup _{\substack{s \geq 0 \\
y \in \mathbf{Y}}}\left\|\mathbf{r}_{\mathbf{0}}(t+s)\right\|+\sup _{\substack{s \geq 0 \\
y \in \mathbf{Y}}}\left\|\mathbf{r}_{\mathbf{1}}(t+s)\right\|
\end{aligned}
$$

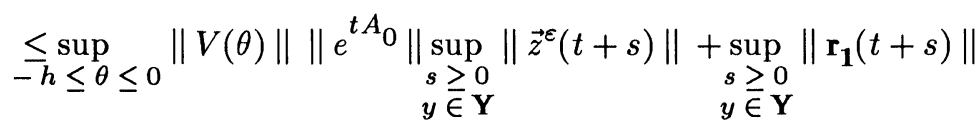

$$
\begin{aligned}
& \leq h_{1} \sup _{\substack{s \geq 0, y \in \mathbf{Y}}}\left(\left|\vec{z}^{\varepsilon}(t+s)-\widetilde{z}^{\varepsilon}(t+s)\right|+\left|\tilde{z}^{\varepsilon}(t+s)\right|\right)+\sup _{\substack{s \geq 0 \\
y \in \mathbf{Y}}}\left\|\mathbf{r}_{\mathbf{1}}(t+s)\right\| \\
& =h_{1} \sup _{\substack{s \geq 0, y \in \mathbf{Y}}}\left(\left|\vec{z}^{\epsilon}(t+s)-\tilde{z}^{\epsilon}(t+s)\right|+\sup _{\substack{s \geq 0 \\
y \in \mathbf{Y}}}\left|\tilde{x}^{\varepsilon}(t \varepsilon+s)\right|\right)+\sup _{\substack{s \geq 0 \\
y \in \mathbf{Y}}}\left\|\mathbf{r}_{\mathbf{1}}(t+s)\right\|,
\end{aligned}
$$

with some positive constant $h_{1}$. Taking into account the definition of the initial condition $\vec{u}$ given by Equation (17) and the formulas in Equations (13), (26) and (38), one can find sufficiently large $A(T)$ as $T \rightarrow \infty$ and infinitesimal $\alpha(\varepsilon)$ as $\varepsilon \rightarrow 0$, such that:

$$
\sup _{\substack{s \geq 0, y \in \mathbf{Y}}} \mathbf{E}_{\varphi, y}^{(s)}\left\{\left\|u_{s+T / \varepsilon}^{\varepsilon}\right\|^{p}\right\} \leq\left(\alpha(\varepsilon) A(T)+\beta e^{-\beta_{1} T}\right)\|\varphi\|^{p}
$$

for any $\varphi \in \mathbf{C}_{n}$ with some positive constant $\beta$. Choosing the numbers $T=\frac{\ln 4 \beta}{\beta_{1}}$ and $\varepsilon_{0}$ such that

$$
\alpha\left(\varepsilon_{0}\right) A\left(\frac{\ln 4 \beta}{\beta}\right)=\frac{1}{4}
$$


this inequality can be rewritten in the form:

$$
\sup _{\substack{s \geq 0, y \in \mathbf{Y}}} \mathbf{E}_{\varphi, y}^{(s)}\left\{\left\|u_{s+T / \varepsilon}^{\varepsilon}\right\|^{p}\right\} \leq \frac{1}{2}\|\varphi\|^{p} .
$$

Next, we apply the Markov property for conditional expectation in the form

$$
\begin{aligned}
& \mathbf{E}_{\varphi, y}^{(s)}\left\{\left\|u_{s+t_{1}+t_{2}}^{\varepsilon}\right\|^{p}\right\} \\
& =\mathbf{E}_{\varphi, y}^{(s)}\left\{\left.\mathbf{E}_{\psi, z}^{(\tau)}\left\{\left\|u_{\tau+t_{2}}^{\varepsilon}\right\|^{p}\right\}\right|_{\tau=t_{1}+s, \psi=u_{s+t_{1}}^{\varepsilon}, z=y_{s+t_{1}}}\right\}
\end{aligned}
$$

This allows us to use the inequality in Equation (39) and to evaluate the second moment of the norm of the solution of Equations (1)-(9) in the recursive form:

$$
\begin{aligned}
\sup _{y \in \mathbf{Y}} \mathbf{E}_{\varphi, y}^{(s)}\left\{\left\{\left\|u_{s_{k+1}}^{\varepsilon}\right\|^{p}\right\}\right. & \left.=\left.\sup _{y \in \mathbf{Y}} \mathbf{E}_{\varphi, y}^{(s)} \mathbf{E}_{\psi, z}^{(\tau)}\left\|u_{\tau+T / \varepsilon}^{\varepsilon}\right\|^{p}\right|_{\tau=s_{k}, \psi=u_{s_{k}}^{\varepsilon}, z=y_{s_{k}}}\right\} \\
& \leq \frac{1}{2} \sup _{y \in \mathbf{Y}} \mathbf{E}_{\varphi, y}^{(s)}\left\{\left\|u_{s_{k}}^{\varepsilon}\right\|^{p}\right\}
\end{aligned}
$$

for any given $s>0, k \in \mathbb{N}$ and $\varphi \in \mathbf{C}_{n}$. Therefore, for $t \in\left[s_{k}, s_{k+1}\right), k \in \mathbb{N}$, the reiteration of the above inequality allows us to write the following inequalities for the second moment of the solution of Equations (1)-(9):

$$
\begin{gathered}
\mathbf{E}_{y, \varphi}^{(s)}\left\{\left|u^{\varepsilon}(s+t / \varepsilon)\right|^{p}\right\} \leq \sup _{s_{k} \leq t<s_{k+1}} \mathbf{E}_{y, \varphi}^{(s)}\left\{\left|u^{\varepsilon}(s+t / \varepsilon)\right|^{p}\right\} \\
\leq c^{p} e^{p c l T} \mathbf{E}_{y, \varphi}^{(s)}\left\{\left\|u_{s_{k}}^{\varepsilon}\right\|^{p}\right\} \leq \frac{c^{p}}{2^{k}} e^{p c l T}\|\varphi\|^{p}
\end{gathered}
$$

or

$$
\mathbf{E}_{\boldsymbol{y}, \varphi}^{(s)}\left\{\left|u^{\varepsilon}(s+t)\right|^{p}\right\} \leq a_{1} e^{-a_{2} t \varepsilon}\|\varphi\|^{p}
$$

with some positive constants $a_{1}, a_{2}$. This completes the proof of our theorem.

\section{Acknowledgements}

This paper is based upon work partly supported by the Hong Kong Research Grant Council under grant no. HKUST 639/95E and by the Latvian Scientific Council under grant no. 96.0540. The authors would like to express their sincere appreciation to the reviewers for their thorough review and their constructive comments and suggestions.

\section{References}

[1] Blankenship, G. and Papanicolaou, G.C., Stability and control of stochastic systems with wide-band noise disturbances I, SIAM J. Appl. Math. 34:3 (1978), 
437-476.

[2] Dynkin, E.B., Markov Processes, Vols. 1 and 2, Springer-Verlag, Berlin 1965.

[3] Halanay, A., On the method of averaging for differential equations with retarded argument, J. Math. Anal. Appl. 14:2 (1966), 70-76.

[4] Hale, J. and Sjord, M., Introduction to Functional Differential Equations, Springer-Verlag, New York-Hong Kong 1993.

[5] Kato, T., Perturbation Theory for Linear Operators, Springer-Verlag, BerlinHeidelberg 1966.

[6] Khas'minskii, R.Z., Stochastic Stability of Differential Equations, Sijthoff and Noordhoff, Alphen aan den Rijn, The Netherlands 1980.

[7] Korolyuk, V.S., Averaging and stability of dynamical systems with rapid Markov switchings, Preprint S-90187, Umea University, Umea, Sweden, February 1991.

[8] Mohammed, S.-E., Stochastic Functional Differential Equations, Pitman, London 1984.

[9] Skorokhod, A.V., Asymptotic Methods of the Theory of Stochastic Differential Equations, AMS, Providence, RI 1989. 


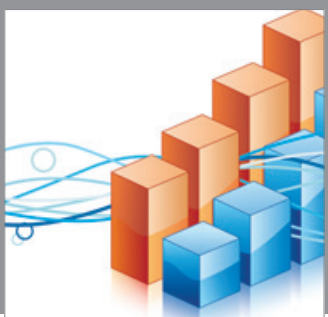

Advances in

Operations Research

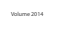

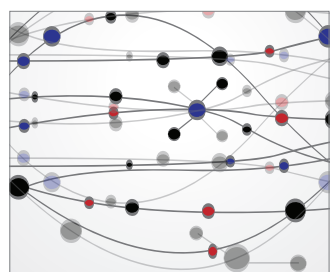

\section{The Scientific} World Journal
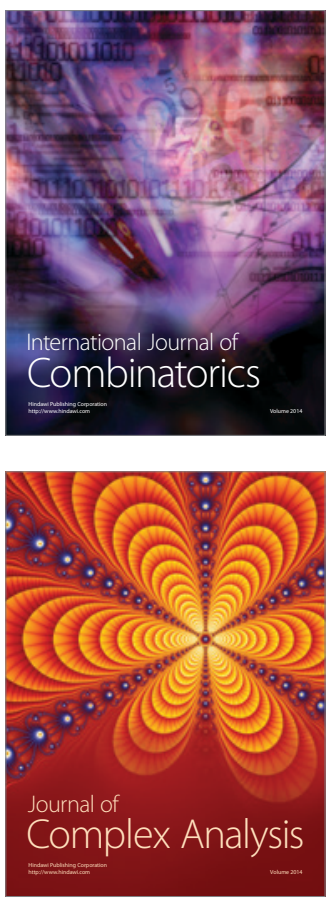

International Journal of

Mathematics and

Mathematical

Sciences
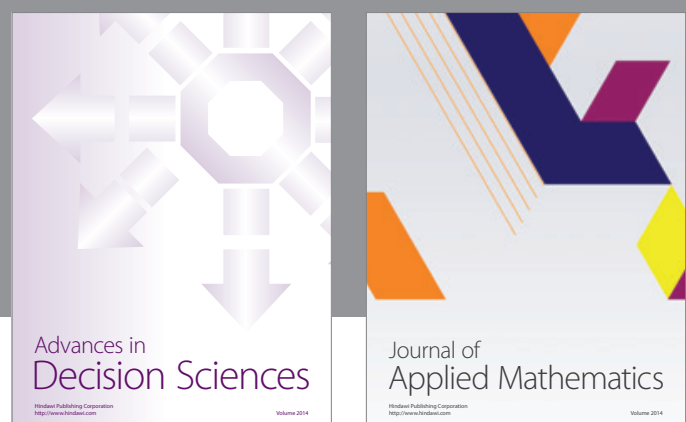

Journal of

Applied Mathematics
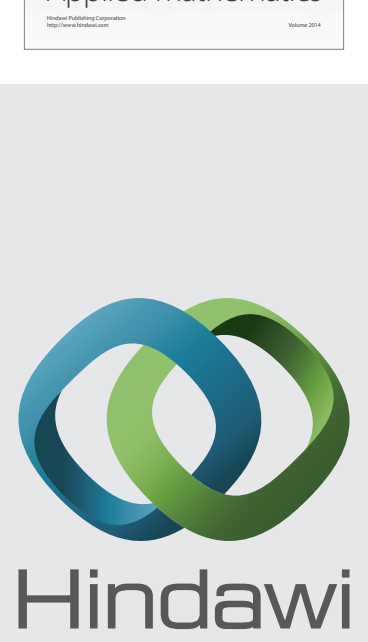

Submit your manuscripts at http://www.hindawi.com
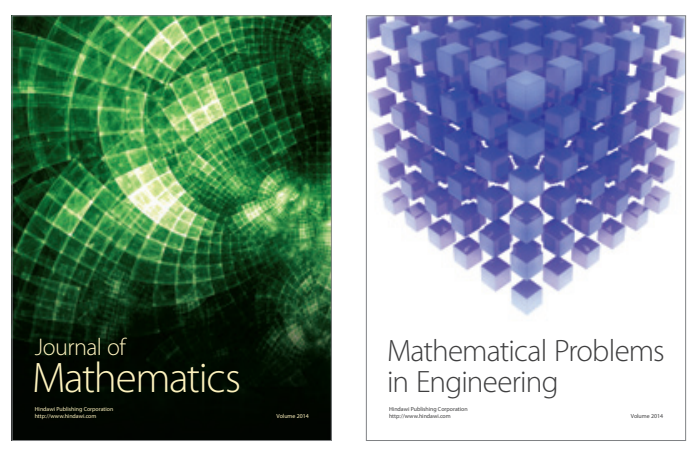

Mathematical Problems in Engineering
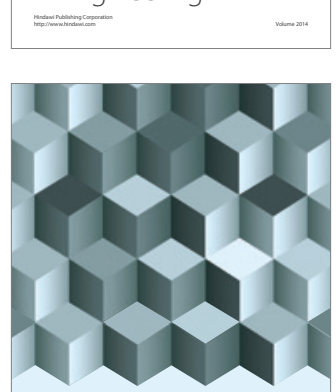

Journal of

Function Spaces
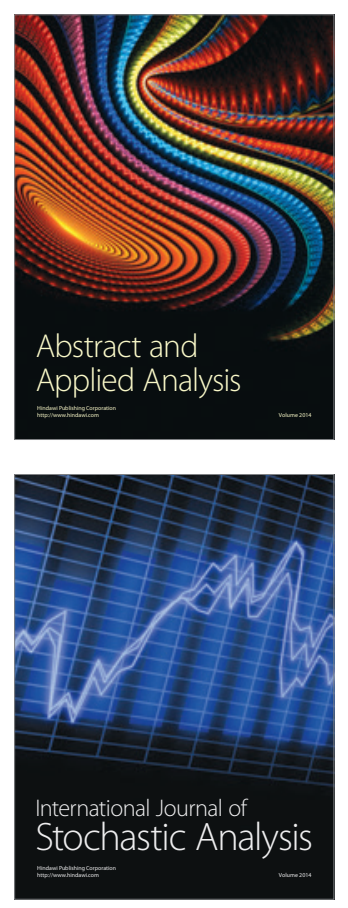

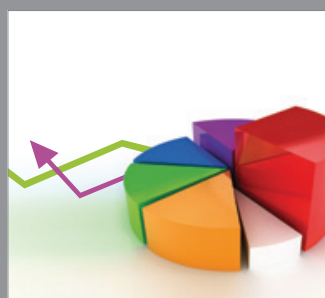

ournal of

Probability and Statistics

Promensencen
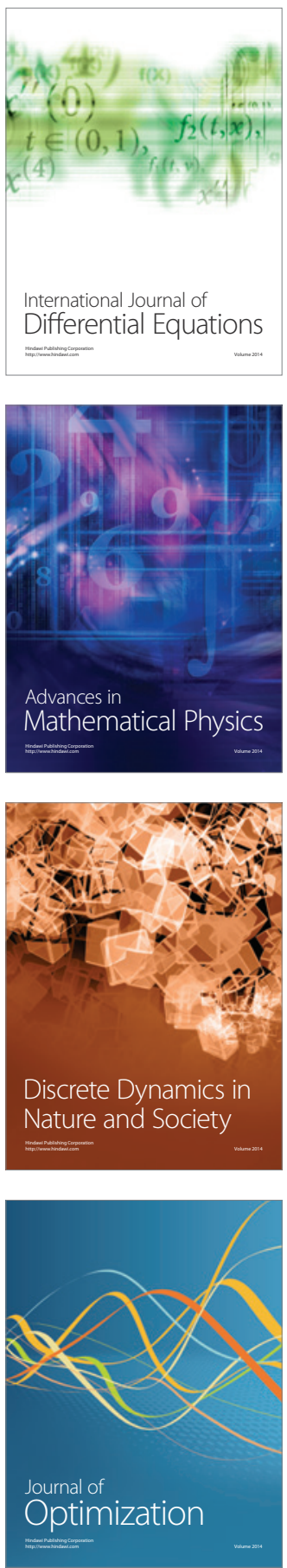